\title{
A new class of Ornstein transformations with singular spectrum
}

\author{
E.H. El Abdalaoui ${ }^{\mathrm{a}, *}$, F. Parreau ${ }^{\mathrm{b}}$, A.A. Prikhod'ko ${ }^{\mathrm{c}}$ \\ a Department of Mathematics, University of Rouen, LMRS, UMR 60 85, avenue de l'Université, BP 12, 76801 Saint Etienne du Rouvray, France \\ ${ }^{\mathrm{b}}$ Department of Mathematics, University Paris 13 LAGA, UMR 7539 CNRS, 99, av. J-B Clément, 93430 Villetaneuse, France \\ c Department of Mathematics, Moscow State University, Russia
}

Received 30 June 2004; received in revised form 5 September 2005; accepted 30 September 2005

Available online 27 March 2006

\begin{abstract}
It is shown that for any family of probability measures in Ornstein type constructions, the corresponding transformation has almost surely a singular spectrum. This is a new generalization of Bourgain's theorem [J. Bourgain, On the spectral type of Ornstein class one transformations, Israel J. Math. 84 (1993) 53-63], same result is proved for Rudolph's construction [D. Rudolph, An example of a measure-preserving map with minimal self-joining and applications, J. Anal. Math. 35 (1979) 97-122].

(c) 2006 Elsevier Masson SAS. All rights reserved.

\section{Résumé}

On montre que pour toute famille de mesures de probabilités dans la construction d'Ornstein, les transformations résultantes ont un spectre presque sûrement singulier. On obtient ainsi une nouvelle généralisation d'un théoréme dû à Bourgain [J. Bourgain, On the spectral type of Ornstein class one transformations, Israel J. Math. 84 (1993) 53-63]. Un résultat similaire est obtenu pour les transformations de Rudolph [D. Rudolph, An example of a measure-preserving map with minimal self-joining and applications, J. Anal. Math. 35 (1979) 97-122].

๑ 2006 Elsevier Masson SAS. All rights reserved.
\end{abstract}

MSC: primary 28D05; secondary 47A35

Keywords: Ornstein transformations; Simple Lebesgue spectrum; Singular spectrum; Generalized Riesz products; Rank one transformations

\section{Introduction}

In this note we investigate the spectral analysis of a generalized class of Ornstein transformations. There are several generalizations of Ornstein transformations. Here we are concerned with arbitrary product probability space associated to random constructions of rank one transformations. Namely, in the Ornstein's construction, the probability space is equipped with the infinite product of uniform probability measures on some finite subsets of $\mathbb{Z}$. Here, the probability space is equipped with the infinite product of probability measures $\left(\xi_{m}\right)_{m \in \mathbb{N}}$ on a family $\left(X_{m}\right)_{m \in \mathbb{N}}$ of finite subsets

\footnotetext{
* Corresponding author.

E-mail addresses: Elhoucein.Elabdalaoui@univ-rouen.fr (E.H. El Abdalaoui), parreau@math.univ-paris13.fr (F. Parreau), apri7@geocities.com (A.A. Prikhod'ko).
} 
of $\mathbb{Z}$. We establish that for any choice of the family $\left(\xi_{m}\right)_{m \in \mathbb{N}}$ the associated Ornstein transformations has almost surely singular spectrum.

Let us recall that Ornstein introduced these transformations in 1967 in [15] and proved that the mixing property occurs almost surely. Until 1991, these transformations which have simple spectrum appeared as a candidate for an affirmative answer to Banach's well-known problem whether a dynamical system $(X, \mathcal{B}, \mu)$ may have simple Lebesgue spectrum. But, in 1991, J. Bourgain in [4], using Riesz products techniques, proved that Ornstein transformations have almost surely singular spectrum. Subsequently, I. Klemes [12], I. Klemes and K. Reinhold [13] obtain that the spectrum of the mixing subclass of staircase transformations of T. Adams [1] and T. Adams and N. Friedman [2] have singular spectrum. They conjectured that rank one transformations always have singular spectrum.

In this paper, using the techniques of J. Bourgain generalized in [8], we extend Bourgain's theorem to the generalized Ornstein transformations associated to a large family of random constructions.

Firstly, we shall recall some basic facts from spectral theory. A nice account can be found in the appendix of [16]. We shall assume that the reader is familiar with the method of cutting and stacking for constructing rank one transformations.

Given $T:(X, \mathcal{B}, \mu) \mapsto(X, \mathcal{B}, \mu)$ a measure preserving invertible transformation and denoting by $U_{T} f$ the operator $U_{T} f(x)=f\left(T^{-1} x\right)$ on $L^{2}(X, \mathcal{B}, \mu)$, recall that to any $f \in L^{2}(X)$ there corresponds a positive measure $\sigma_{f}$ on $\mathbb{T}$, the unit circle, defined by $\hat{\sigma}_{f}(n)=\left\langle U_{T}^{n} f, f\right\rangle$.

Definition 1.1. The maximal spectral type of $T$ is the equivalence class of Borel measures $\sigma$ on $\mathbb{T}$ (under the equivalence relation $\mu_{1} \sim \mu_{2}$ if and only if $\mu_{1} \ll \mu_{2}$ and $\left.\mu_{2} \ll \mu_{1}\right)$, such that $\sigma_{f} \ll \sigma$ for all $f \in L^{2}(X)$ and if $v$ is another measure for which $\sigma_{f} \ll v$ for all $f \in L^{2}(X)$ then $\sigma \ll v$.

There exists a Borel measure $\sigma=\sigma_{f}$ for some $f \in L^{2}(X)$, such that $\sigma$ is in the equivalence class defining the maximal spectral type of $T$. By abuse of notation, we will call this measure the maximal spectral type measure. The reduced maximal type $\sigma_{0}$ is the maximal spectral type of $U_{T}$ on $L_{0}^{2}(X) \stackrel{\text { def }}{=}\left\{f \in L^{2}(X): \int f \mathrm{~d} \mu=0\right\}$. The spectrum of $T$ is said to be discrete (resp. continuous, resp. singular, resp. absolutely continuous, resp. Lebesgue) if $\sigma_{0}$ is discrete (resp. continuous, resp. singular, resp. absolutely continuous with respect to the Lebesgue measure or equivalent to the Lebesgue measure). We write

$$
Z(h) \stackrel{\text { def }}{=} \overline{\operatorname{span}\left\{U_{T}^{n} h, n \in \mathbb{Z}\right\}} \text {. }
$$

$T$ is said to have simple spectrum, if there exists $h \in L^{2}(X)$ such that

$$
Z(h)=L^{2}(X) .
$$

\section{Rank one transformation by construction}

Using the cutting and stacking method described in [10,11], one defines inductively a family of measure preserving transformations, called rank one transformations, as follows.

Let $B_{0}$ be the unit interval equipped with the Lebesgue measure. At stage one we divide $B_{0}$ into $p_{0}$ equal parts, add spacers and form a stack of height $h_{1}$ in the usual fashion. At the $k$ th stage we divide the stack obtained at the $(k-1)$ th stage into $p_{k-1}$ equal columns, add spacers and obtain a new stack of height $h_{k}$. If during the $k$ th stage of our construction the number of spacers put above the $j$ th column of the $(k-1)$ th stack is $a_{j}^{(k-1)}, 0 \leqslant a_{j}^{(k-1)}<\infty$, $1 \leqslant j \leqslant p_{k}$, then we have (see Fig. 1)

$$
\begin{aligned}
& h_{k}=p_{k-1} h_{k-1}+\sum_{j=1}^{p_{k-1}} a_{j}^{(k-1)}, \quad \forall k \geqslant 1, \\
& h_{0}=1 .
\end{aligned}
$$

Proceeding thus we get a rank one transformation $T$ on a certain measure space $(X, \mathcal{B}, v)$ which may be finite or $\sigma$-finite depending on the number of spacers added. 


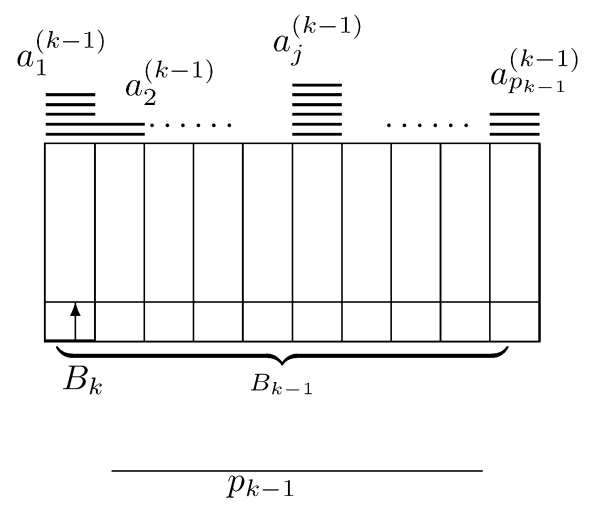

Fig. 1. $k$ th-tower.

The construction of any rank one transformation thus needs two parameters $\left(p_{k}\right)_{k=0}^{\infty}$ (cutting parameter) and $\left(\left(a_{j}^{(k)}\right)_{j=1}^{p_{k}}\right)_{k=0}^{\infty}$ (spacers parameter). We put

$$
T \stackrel{\text { def }}{=} T_{\left(p_{k},\left(a_{j}^{(k)}\right)_{j=1}^{p_{k}}\right)_{k=0}^{\infty}}
$$

In $[4,5]$ and [13] it is proved that up to some discrete measure, the spectral type of this transformation is given by

$$
\mathrm{d} \sigma=W^{*} \lim \prod_{k=1}^{n}\left|P_{k}\right|^{2} \mathrm{~d} \lambda
$$

where

$$
P_{k}(z)=\frac{1}{\sqrt{p_{k}}}\left(\sum_{j=0}^{p_{k}-1} z^{-\left(j h_{k}+\sum_{i=1}^{j} a_{i}^{(k)}\right)}\right) .
$$

$\lambda$ denotes the normalized Lebesgue measure on torus $\mathbb{T}$ and $W^{*} \lim$ denotes weak*limit in the space of bounded Borel measures on $\mathbb{T}$. The polynomials $P_{k}$ appear naturally from the induction relation between the bases $B_{k}$. Indeed

$$
\begin{aligned}
& B_{k}=\bigcup_{j=0}^{p_{k}-1} T^{h_{k}+s_{k}(j)} B_{k+1}, \\
& v\left(B_{k}\right)=p_{k} v\left(B_{k+1}\right),
\end{aligned}
$$

where $s_{k}(n)=a_{1}^{(k)}+\cdots+a_{n}^{(k)}$ and $s_{k}(0)=0$.

Put

$$
f_{k}=\frac{1}{\sqrt{v\left(B_{k}\right)}} \chi_{B_{k}}
$$

that is the indicator function of the $k$ th-base normalized in the $L^{2}$-norm. So

$$
f_{k}=P_{k}\left(U_{T}\right) f_{k+1},
$$

It follows that

$$
\mathrm{d} \sigma_{k}=\left|P_{k}\right|^{2} \mathrm{~d} \sigma_{k+1}=\cdots=\prod_{j=0}^{m-1}\left|P_{k+j}\right|^{2} \mathrm{~d} \sigma_{k+m},
$$

where $\sigma_{p}$ is the spectral measure of $f_{p}, p \geqslant 0$. 


\section{Generalized Ornstein's class of transformations}

In Ornstein's construction, the $p_{k}$ 's are rapidly increasing, and the number of spacers, $a_{i}^{(k)}, 1 \leqslant i \leqslant p_{k}-1$, are chosen randomly. This may be organized in different ways as pointed by J. Bourgain in [4]. Here, We suppose given $\left(t_{k}\right),\left(p_{k}\right)$ a sequence of positive integers and $\left(\xi_{k}\right)$ a sequence of probability measure such that the support of each $\xi_{k}$ is a subset of $X_{k}=\left\{-\frac{t_{k}}{2}, \cdots, \frac{t_{k}}{2}\right\}$. We choose now independently, according to $\xi_{k}$ the numbers $\left(x_{k, i}\right)_{i=1}^{p_{k}-1}$, and $x_{k, p_{k}}$ is chosen deterministically in $\mathbb{N}$. We put, for $1 \leqslant i \leqslant p_{k}$,

$$
a_{i}^{(k)}=t_{k}+x_{k, i}-x_{k, i-1}, \quad \text { with } x_{k, 0}=0 .
$$

It follows

$$
h_{k+1}=p_{k}\left(h_{k}+t_{k}\right)+x_{k, p_{k}} .
$$

So the deterministic sequences of positive integers $\left(p_{k}\right)_{k=0}^{\infty},\left(t_{k}\right)_{k=0}^{\infty}$ and $\left(x_{k, p_{k}}\right)_{k=0}^{\infty}$ determine completely the sequence of heights $\left(h_{k}\right)_{k=0}^{\infty}$. The total measure of the resulting measure space is finite if

$$
\sum_{k=0}^{\infty} \frac{t_{k}}{h_{k}}+\sum_{k=0}^{\infty} \frac{x_{k, p_{k}}}{p_{k} h_{k}}<\infty .
$$

We will assume that this requirement is satisfied.

We thus have a probability space of Ornstein transformations $\Omega=\prod_{l=0}^{\infty} X_{l}^{p_{l}-1}$ equipped with the natural probability measure $\mathbb{P} \stackrel{\text { def }}{=} \bigotimes_{l=1}^{\infty} P_{l}$, where $P_{l} \stackrel{\text { def }}{=} \bigotimes_{j=1}^{p_{l}-1} \xi_{l} ; \xi_{l}$ is the probability measure on $X_{l}$. We denote this space by $(\Omega, \mathcal{A}, \mathbb{P})$. So $x_{k, i}, 1 \leqslant i \leqslant p_{k}-1$, is the projection from $\Omega$ onto the $i$ th co-ordinate space of $\Omega_{k} \stackrel{\text { def }}{=} X_{k}^{p_{k}-1}$, $1 \leqslant i \leqslant p_{k}-1$. Naturally each point $\omega=\left(\omega_{k}=\left(x_{k, i}(\omega)\right)_{i=1}^{p_{k}-1}\right)_{k=0}^{\infty}$ in $\Omega$ defines the spacers and therefore a rank one transformation $T_{\omega, x}$, where $x=\left(x_{k, p_{k}}\right)$.

The definition above gives a more general definition of random construction due to Ornstein. In the particular case of Ornstein's transformations constructed in [15], $t_{k}=h_{k-1}, \xi_{k}$ is the uniform distribution on $X_{k}$ and $p_{k} \gg h_{k-1}$.

We recall that Ornstein in [15] proved that there exist a sequence $\left(p_{k}, x_{k, p_{k}}\right)_{k \in \mathbb{N}}$ such that, $T_{\omega, x}$ is almost surely mixing. Later in [17], Prikhod'ko obtain the same result for some special choice of the sequence of the distribution $\left(\xi_{m}\right)$ and recently, using the idea of D. Creutz and C.E. Silva [6] one can extend this result to a large family of the probability measure associated to Ornstein construction. In our general construction, according to (2.1) the spectral type of each $T_{\omega}$, up to a discrete measure, is given by

$$
\sigma_{T_{\omega}}=\sigma_{\chi_{B_{0}}}^{(\omega)}=\sigma^{(\omega)}=W^{*} \lim \prod_{l=1}^{N} \frac{1}{p_{l}}\left|\sum_{p=0}^{p_{l}-1} z^{p\left(h_{l}+t_{l}\right)+x_{l, p}}\right|^{2} \mathrm{~d} \lambda .
$$

In the case of Rudolph construction, we choose independently, using the uniform distribution on the set $\left\{1, \ldots, t_{k}\right\}$ the numbers $\left(x_{k, i}\right)_{i=1}^{p_{k}}$. We put, for $1 \leqslant i \leqslant p_{k}-1$,

$$
\begin{aligned}
& a_{i}^{(k)}=t_{k}+2+x_{k, i+1}-x_{k, i}, \\
& a_{p_{k}}^{(k)}=t_{k}+1-x_{k, p_{k}} .
\end{aligned}
$$

We define $a_{-1}^{(k)}$ as de number of spacers we stack below the first column. We put

$$
a_{-1}^{(k)}=x_{k, 1}+1 \text {. }
$$

One sees that

$$
h_{k+1}=p_{k}\left(h_{k}+t_{k}+2\right) \text {. }
$$

For Rudolph construction, according to (2.1) the spectral type of each $T_{\omega}$, up to a discrete measure, is given by

$$
\sigma_{T_{\omega}}=\sigma_{\chi_{B_{0}}}^{(\omega)}=\sigma^{(\omega)}=W^{*} \lim \prod_{l=1}^{N} \frac{1}{p_{l}}\left|\sum_{p=0}^{p_{l}-1} z^{p\left(h_{l}+t_{l}+2\right)+x_{l, p+1}-x_{l, 1}}\right|^{2} \mathrm{~d} \lambda .
$$

With the above notation, we state our main result. 
Theorem 3.1. For every choice of $\left(p_{k}\right),\left(t_{k}\right),\left(x_{k, p_{k}}\right)$ and for any family of probability measures $\xi_{k}$ on $X_{k}=$ $\left\{-t_{k}, \ldots, t_{k}\right\}$ of $\mathbb{Z}, k \in \mathbb{N}^{*}$, the associated generalized Ornstein transformations has almost surely singular spectrum, i.e.

$$
\mathbb{P}\left\{\omega: \sigma^{(\omega)} \perp \lambda\right\}=1,
$$

where $\mathbb{P} \stackrel{\text { def }}{=} \bigotimes_{l=0}^{\infty} \bigotimes_{j=1}^{p_{l}-1} \xi_{l}$ is the probability measure on $\Omega=\prod_{l=0}^{\infty} X_{l}^{p_{l}-1}, X_{l}$ is finite subset of $\mathbb{Z}$.

Before we proceed to the proof, we remark that it is an easy exercise to see that the spectrum of Ornstein's transformation is always singular if the cutting parameter $p_{k}$ is bounded. In fact, Klemes and Reinhold proved that if $\sum_{k=0}^{\infty} \frac{1}{p_{k}{ }^{2}}=\infty$ then the associated rank one transformation is singular. Henceforth, we assume that the series $\sum_{k=0}^{\infty} \frac{1}{p_{k}^{2}}$ converges.

We shall adapt Bourgain's proof. For that, we need a local version of the singularity criterion used by Bourgain. Let $F$ be a Borel set then with the above notation, we will state local singularity criterion in the following form.

Theorem 3.2 ((Local Singularity Criterion)). The following are equivalent

(i) $\sigma_{F} \perp \lambda$, where $\sigma_{F}=\chi_{F} \mathrm{~d} \sigma, \chi_{F}$ is a indicator function of $F$.

(ii) $\int_{F} \prod_{l=1}^{n}\left|P_{l}(z)\right| \mathrm{d} \lambda \underset{n \rightarrow \infty}{\longrightarrow} 0$.

(iii) $\inf \left\{\int_{F} \prod_{l=1}^{k}\left|P_{n_{l}}(z)\right| \mathrm{d} \lambda, k \in \mathbb{N}, n_{1}<n_{2}<\cdots<n_{k}\right\}=0$.

One can adapt the proof of Theorem 4.3 in [13], or in [8,14].

Now, using Lebesgue's dominated convergence theorem and the local singularity criterion, we obtain

\section{Proposition 3.3. The following are equivalent}

(i) $\sigma_{F}^{(\omega)} \perp \lambda \mathbb{P}$ a.s.

(ii) $\int_{F} \prod_{l=1}^{n}\left|P_{l}(z)\right| \mathrm{d} \lambda \mathrm{d} \mathbb{P} \underset{n \rightarrow \infty}{\longrightarrow} 0$.

(iii) $\inf \left\{\iint_{F} \prod_{l=1}^{k}\left|P_{n_{l}}(z)\right| \mathrm{d} \lambda \mathrm{d} \mathbb{P}, k \in \mathbb{N}, n_{1}<n_{2}<\cdots<n_{k}\right\}=0$.

Fix some subsequence $\mathcal{N}=\left\{n_{1}<n_{2}<\cdots<n_{k}\right\}, k \in \mathbb{N}, m>n_{k}$ and put

$$
Q(z)=\prod_{i=1}^{k}\left|P_{n_{i}}(z)\right|
$$

Following [4] (see also [12] or [9]), we have, for $m>n_{k}$.

\section{Lemma 3.4.}

$$
\int_{F} Q\left|P_{m}\right| \mathrm{d} \lambda \leqslant \frac{1}{2}\left(\int_{F} Q \mathrm{~d} \lambda+\int_{F} Q\left|P_{m}\right|^{2} \mathrm{~d} \lambda\right)-\frac{1}{8}\left(\left.\int_{F} Q|| P_{m}\right|^{2}-1 \mid \mathrm{d} \lambda\right)^{2} .
$$

Now, we assume that $F$ is a closed set, it follows

\section{Lemma 3.5.}

$$
\limsup _{m \rightarrow \infty} \int_{F} Q\left|P_{m}(z)\right|^{2} \mathrm{~d} \lambda(z) \leqslant \int_{F} Q \mathrm{~d} \lambda(z) .
$$


Proof. The sequence of probability measures $\left|P_{m}(z)\right|^{2} \mathrm{~d} \lambda(z)$ converges weakly to the Lebesgue measure. Then the lemma follows from the classical portmanteau theorem. ${ }^{1}$

From Lemmas 3.4 and 3.5 we get the following

\section{Lemma 3.6.}

$$
\liminf \iint_{F} Q\left|P_{m}\right| \mathrm{d} \lambda \mathrm{d} \mathbb{P} \leqslant \iint_{F} Q \mathrm{~d} \lambda \mathrm{d} \mathbb{P}-\frac{1}{8}\left(\left.\lim \sup \iint_{F} Q|| P_{m}\right|^{2}-1 \mid \mathrm{d} \lambda \mathrm{d} \mathbb{P}\right)^{2} .
$$

Clearly, we need to estimate the quantity

$$
\left.\iint_{F} Q|| P_{m}(z)\right|^{2}-1 \mid \mathrm{d} \lambda(z) \mathrm{d} \mathbb{P} .
$$

For that, following Bourgain we shall prove the following

Proposition 3.7. There exists an absolute constant $K>0$ such that

$$
\left.\limsup \iint_{F} Q|| P_{m}\right|^{2}-1 \mid \mathrm{d} \lambda \mathrm{d} \mathbb{P} \geqslant K\left(\iint_{F} Q \mathrm{~d} \lambda \mathrm{d} \mathbb{P}-\liminf \iint_{F} Q(z) \phi_{m}(z) \mathrm{d} \lambda \mathrm{d} \mathbb{P}\right)^{2},
$$

where $\phi_{m}(z)=\left|\sum_{p=-t_{m} / 2}^{p=t_{m} / 2} \xi_{m}(p) z^{p}\right|^{2}, z \in \mathbb{T}$.

We shall give the proof of Proposition 3.7 in the following section.

\section{Khintchine-Bonami inequality}

Fix $z \in \mathbb{T}$ and $m \in \mathbb{N}^{*}$. Define $\tau$ and $\left(\tau_{p}\right)_{p=1}^{p_{m}-1}$ by

$$
\begin{aligned}
\tau: \mathbb{Z} & \longrightarrow \mathbb{T} \\
s & \longmapsto z^{s} .
\end{aligned}
$$

$\tau_{p}$ is given by $\tau_{p}=\tau \circ x_{m, p}, x_{m, p}$ is the $p$ th projection on $\Omega_{m}=X_{m}^{p_{m}-1}$. So

$$
\left|P_{m}(z)\right|^{2}-1=\sum_{p \neq q, p \neq 0, q \neq 0} a_{p q} \tau_{p}(\omega) \overline{\tau_{q}}(\omega)+2 \operatorname{Re}\left\{\frac{1}{p_{m}} \sum_{p \neq 0} z^{p} \tau_{p}(\omega)\right\} .
$$

Where

$$
a_{p q}=\frac{z^{(p-q)\left(h_{m}+t_{m}\right)}}{p_{m}} .
$$

The random variables $\left(\tau_{p}\right)_{p=1}^{p_{m}-1}$ are independent. Put

$$
\tau_{p}^{\circ}=\tau_{p}-\int \tau_{p} \mathrm{~d} \mathbb{P}, \quad p=1, \ldots, p_{m}-1
$$

and write

$$
\begin{aligned}
& \sum_{p \neq q, p \neq 0, q \neq 0} a_{p q} \tau_{p} \overline{\tau_{q}} \\
= & \left(\sum a_{p q}\right)\left|\int \tau_{1} \mathrm{~d} \mathbb{P}\right|^{2}+\sum a_{p q}\left(\left(\int \overline{\tau_{1}} \mathrm{~d} \mathbb{P}\right) \tau_{p}^{\circ}+\left(\int \tau_{1} \mathrm{~d} \mathbb{P}\right) \overline{\tau_{q}^{\circ}}\right)+\sum a_{p q} \tau_{p}^{\circ} \overline{\tau_{q}^{\circ}} .
\end{aligned}
$$

\footnotetext{
1 See for example [7].
} 
Now, using the same arguments as J. Bourgain, let us consider a random sign $\varepsilon=\left\{\varepsilon_{1}, \ldots, \varepsilon_{p_{m}-1}\right\} \in\{-1,1\}^{p_{m}-1}$, and the probability space

$$
Z_{m}=\Omega_{m} \times\{-1,1\}^{p_{m}-1}, \quad \text { where } \Omega_{m}=\left\{-\frac{t_{m}}{2}, \ldots, \frac{t_{m}}{2}\right\}^{p_{m}-1} .
$$

Taking the conditional expectation of the following quantity

$$
\sum_{p \neq q, p \neq 0, q \neq 0} a_{p q}\left(\int \overline{\tau_{1}} \mathrm{~d} \mathbb{P} \tau_{p}^{\circ}+\int \tau_{1} \mathrm{~d} \mathbb{P} \overline{\tau_{q}^{\circ}}\right)+\sum_{p \neq q, p \neq 0, q \neq 0} a_{p q} \tau_{p}^{\circ} \overline{\tau_{q}^{\circ}}
$$

with respect to the $\sigma$-algebra $\mathcal{B}_{\varepsilon}$ given by the cylinders sets $A(I, x)$ where $I \subset\left\{1, \ldots, p_{m}-1\right\}, x \in \Omega_{m}$ and

$$
A(I, x)=\prod_{i \in I}\left\{x_{i}\right\} \times\left\{-\frac{t_{m}}{2}, \ldots, \frac{t_{m}}{2}\right\}^{\left|I^{c}\right|} \times\{1\}^{|I|} \times\{-1\}^{\left|I^{c}\right|}
$$

( $I$ corresponds to $\varepsilon_{i}=1, \forall i \in I$ and $\varepsilon_{i}=-1, \forall i \notin I$ ), in other words, taking conditional expectation with respect to the random variables $\tau_{p}$ for which $\varepsilon_{p}=1$, one finds the following polynomial expression in $\varepsilon$ of degree 2

$$
\sum a_{p q}\left(\frac{1+\varepsilon_{p}}{2} \int \overline{\tau_{1}} \mathrm{~d} \mathbb{P} \tau_{p}^{\circ}+\frac{1+\varepsilon_{q}}{2} \int \tau_{1} \mathrm{~d} \mathbb{P} \overline{\tau_{q}^{\circ}}\right)+\sum a_{p q} \frac{1+\varepsilon_{p}}{2} \frac{1+\varepsilon_{q}}{2} \tau_{p}^{\circ} \overline{\tau_{q}^{\circ}} .
$$

So

$$
\left.\int|| P_{m}(z)\right|^{2}-1\left|\mathrm{~d} \mathbb{P}=\iint \mathbb{E}\left(\left.|| P_{m}(z)\right|^{2}-\left.1\right|_{\mid \mathcal{B}_{\varepsilon}}\right) \mathrm{d} \mathbb{P} \mathrm{d} \varepsilon \geqslant \iint\right| \mathbb{E}\left(\left|P_{m}(z)\right|^{2}-1_{\mid \mathcal{B}_{\varepsilon}}\right) \mid \mathrm{d} \mathbb{P} \mathrm{d} \varepsilon .
$$

It follows, by the Khintchine-Bonami inequality, ${ }^{2}$ [3], that there exists a positive constant $K$ such that

$$
\begin{aligned}
\iint\left|\mathbb{E}\left(\left|P_{m}(z)\right|^{2}-1_{\mid \mathcal{B}_{\varepsilon}}\right]\right| \mathrm{d} \mathbb{P} \mathrm{d} \varepsilon & \geqslant K \int\left(\int\left|\mathbb{E}\left(\left|P_{m}(z)\right|^{2}-1_{\mid \mathcal{B}_{\varepsilon}}\right]\right|^{2} \mathrm{~d} \varepsilon\right)^{1 / 2} \mathrm{~d} \mathbb{P} \\
& =K \int\left(\sum_{p \neq q}\left|a_{p q}(z) \tau_{p}^{\circ}(z) \overline{\tau_{q}^{\circ}(z)}\right|^{2}\right)^{1 / 2} \mathrm{~d} \mathbb{P} .
\end{aligned}
$$

But all these random variables are bounded by 2 . Hence

$$
\begin{aligned}
\left.\int|| P_{m}(z)\right|^{2}-1 \mid \mathrm{d} \mathbb{P} & \geqslant K^{\prime} \int \frac{1}{p_{m}^{2}} \sum\left|\tau_{p}^{\circ}(z) \overline{\tau_{q}^{\circ}(z)}\right|^{2} \mathrm{~d} \mathbb{P} \\
& =K^{\prime} \frac{1}{p_{m}^{2}} \sum\left(\int\left|\tau_{p}^{\circ}(z)\right|^{2} \mathrm{~d} \mathbb{P}\right)^{2} \\
& =K^{\prime} \frac{\left(p_{m}-1\right)\left(p_{m}-2\right)}{p_{m}^{2}}\left(\int\left|\tau_{1}^{\circ}(z)\right|^{2} \mathrm{~d} \mathbb{P}\right)^{2}
\end{aligned}
$$

Since

$$
\int\left|\tau_{1}^{\circ}(z)\right|^{2} \mathrm{~d} \mathbb{P}=\operatorname{var}\left(\tau_{1}(z)\right)=1-\left|\sum_{s=-t_{m} / 2}^{t_{m} / 2} \xi_{m}(s) z^{s}\right|^{2}=1-\phi_{m}(z) .
$$

Now, combined (4.6) with (4.7) to obtain

$$
\left.\int|| P_{m}(z)\right|^{2}-1 \mid \mathrm{d} \mathbb{P} \geqslant K^{\prime} \frac{\left(p_{m}-1\right)\left(p_{m}-2\right)}{p_{m}^{2}}\left(1-\left|\sum_{s=-t_{m} / 2}^{t_{m} / 2} \xi_{m}(s) z^{s}\right|^{2}\right)^{2} .
$$

\footnotetext{
2 One can extend easily this inequality to bounded sequences of independent real random variables, with vanishing expectation.
} 
Finally, multiply (4.8) by

$$
\int \prod_{j \in \mathcal{N}}\left|P_{j}(z)\right| \mathrm{d} \mathbb{P} \text {. }
$$

Using the independence of (4.9) and $\left.|1-| P_{m}(z)\right|^{2} \mid$. Integrating over $F$ with respect to the Lebesgue measure to get

$$
\left.\iint_{\Omega} Q|| P_{m}\right|^{2}-1 \mid \mathrm{d} \mathbb{P} \mathrm{d} \lambda \geqslant K^{\prime} \frac{\left(P_{m}-1\right)\left(P_{m}-2\right)}{P_{m}^{2}} \iint_{\Omega F}\left(1-\phi_{m}(t)\right) \mathrm{d} \mathbb{P} \mathrm{d} \lambda .
$$

Apply Cauchy-Schwarz inequality to obtain

$$
\begin{aligned}
\iint_{\Omega F} Q\left(1-\phi_{m}(z)\right) \mathrm{d} \lambda \mathrm{d} \mathbb{P} & \leqslant\left(\iint_{\Omega F} Q \mathrm{~d} \lambda \mathrm{d} \mathbb{P}\right)^{1 / 2}\left(\iint_{\Omega F} Q\left(1-\phi_{m}(z)\right)^{2} \mathrm{~d} \lambda \mathrm{d} \mathbb{P}\right)^{1 / 2} \\
& \leqslant\left(\iint_{\Omega F} Q\left(1-\phi_{m}(z)\right)^{2} \mathrm{~d} \lambda \mathrm{d} \mathbb{P}\right)^{1 / 2} .
\end{aligned}
$$

Combine (4.10) and (4.11) and take lim inf to finish the proof of Proposition 3.7.

Now, passing to a subsequence we may assume that $\phi_{m}$ converge weakly in $L^{2}(\lambda)$ to some function $\phi$ in $L^{2}(\lambda)$. Then,

$$
\hat{\phi}(n)=\lim _{m \longrightarrow \infty} \widehat{\phi_{m}}(n) \geqslant 0 \quad \text { for any } n \in \mathbb{Z},
$$

and

$$
\sum_{n} \hat{\phi}(n) \leqslant 1
$$

Hence, the Fourier series of $\phi$ converges absolutely. We still denoted by

$$
\phi(z)=\sum_{n} \hat{\phi}(n) z^{n}
$$

In particular $\phi$ is a continuous function. We deduce that the set $\{\phi(z)=1\}$ is either the torus or a finite subgroup of the torus.

Remark 4.1. It is any easy exercise to see that if the set $\{\phi=1\}$ is not a null set with respect to Lebesgue measure then, for any $z \in \mathbb{T}, \phi(z)=1$. In fact,

$$
\phi(z)=1 \text { everywhere } \quad \text { iff } \widehat{\phi_{m}}(0)=\sum \xi_{m}(s)^{2} \underset{m \rightarrow \infty}{\longrightarrow} 1 \text { hence iff } \max _{s \in X_{m}} \xi_{m}(s) \underset{m \rightarrow \infty}{\longrightarrow} 1 .
$$

\section{On the Ornstein probability space for which $\underline{\lim } \max _{s \in X_{m}} \xi_{m}(s)<1$}

In this section, we assume that $\underline{\lim } \max _{s \in X_{m}} \xi_{m}(s)<1$. So, we may choose a subsequence of $\phi_{m}$ which converge in weak* to $\phi$. Let $\varepsilon>0$,

$$
F_{\varepsilon}=\{z \in \mathbb{T}: 1-\phi(z) \geqslant \varepsilon\} .
$$

$F_{\varepsilon}$ is a closed set and we have also the following proposition

Proposition 5.1. There exists an absolute constant $K>0$ such that

$$
\underline{\lim } \iint_{F_{\varepsilon}} Q \|\left. P_{m}(z)\right|^{2}-1 \mid \mathrm{d} \lambda \mathbb{P} \geqslant K \varepsilon^{2}\left(\iint_{F_{\varepsilon}} Q \mathrm{~d} \lambda \mathbb{P}\right)^{2} .
$$


Proof. Apply Proposition 3.7 to get that there exists an constant $K>0$ for which

$$
\begin{aligned}
\liminf \iint_{F_{\varepsilon}} Q \|\left. P_{m}(z)\right|^{2}-1 \mid \mathrm{d} \lambda \mathbb{P} & \geqslant K\left(\iint_{F} Q \mathrm{~d} \lambda \mathrm{d} \mathbb{P}-\varlimsup \iint_{F} Q(z)\left|\sum_{p=-t_{m} / 2}^{p=t_{m} / 2} \xi_{m}(p) z^{p}\right|^{2} \lambda \mathrm{d} \mathbb{P}\right)^{2} \\
& \geqslant K\left(\iint_{F_{\varepsilon}} Q(1-\phi(z)) \lambda \mathrm{d} \mathbb{P}\right)^{2} \geqslant K \varepsilon^{2}\left(\iiint_{F_{\varepsilon}} Q \lambda \mathrm{d} \mathbb{P}\right)^{2} .
\end{aligned}
$$

The proof of the proposition is complete.

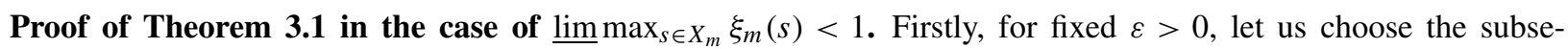
quence $\mathcal{N}$. From Propositions 3.6. and 5.1. one can write

$$
\varlimsup \iint_{F_{\varepsilon}} Q\left|P_{m}(z)\right| \mathrm{d} \lambda(z) \mathrm{d} \mathbb{P} \leqslant \iint_{F_{\varepsilon}} Q-\frac{1}{8} K^{2} \varepsilon^{4}\left(\iint_{F_{\varepsilon}} Q \mathrm{~d} \lambda \mathrm{d} \mathbb{P}\right)^{4},
$$

and from this last inequality we shall construct $\mathcal{N}$. In fact, suppose we have chosen the $k$ first elements of the subsequence $\mathcal{N}$. We wish to define the $(k+1)$ th element. Let $m>n_{k}$ such that

$$
\iint_{F_{\varepsilon}} Q\left|P_{m}(z)\right| \mathrm{d} \lambda(z) \mathrm{d} \mathbb{P} \leqslant \iint_{F_{\varepsilon}} Q \mathrm{~d} \lambda \mathrm{d} \mathbb{P}-\frac{1}{8} K^{2} \varepsilon^{4}\left(\iint_{F_{\varepsilon}} Q \mathrm{~d} \lambda \mathrm{d} \mathbb{P}\right)^{4},
$$

and put $n_{k+1}=m$. It follows that the elements of the subsequence $\mathcal{N}$ verify

$$
\iint_{F_{\varepsilon}} \prod_{i=1}^{k+1}\left|P_{n_{i}}(z)\right| \mathrm{d} \lambda \mathrm{d} \mathbb{P} \leqslant \iint_{F_{\varepsilon}} \prod_{i=1}^{k}\left|P_{n_{i}}(z)\right| \mathrm{d} \lambda \mathrm{d} \mathbb{P}-\frac{1}{8} K^{2} \varepsilon^{4}\left(\iint \prod_{F_{\varepsilon}}^{k}\left|P_{n_{i}}(z)\right| \mathrm{d} \lambda \mathrm{d} \mathbb{P}\right)^{4} .
$$

We deduce that the sequence $\left(\iint_{F_{\varepsilon}} \prod_{i=1}^{k}\left|P_{n_{i}}(z)\right| \mathrm{d} \lambda \mathbb{P}\right)_{k \geqslant 1}$ is decreasing and converges to the limit $l_{\varepsilon}$ which verifies

$$
l_{\varepsilon} \leqslant l_{\varepsilon}-\frac{1}{8} K^{2} \varepsilon^{4} l_{\varepsilon}^{4}
$$

and this implies that $l_{\varepsilon}=0$. Hence, $\sigma_{F_{\varepsilon}}^{(\omega)}$ is singular. But, $\bigsqcup_{\varepsilon>0, \varepsilon \in \mathbb{Q}} F_{\varepsilon}=\{1-\phi \neq 0\}=\{z: \phi(z) \neq 1\}$ is a conull set with respect to the Lebesgue measure. This completes the proof of Theorem 3.1 when $\underline{\lim } \max _{s \in X_{m}} \xi_{m}(s)<1$.

\section{On the Ornstein probability spaces for which $\lim \max _{s \in X_{m}} \xi_{m}(s)=1$}

We have the following

\section{Lemma 6.1.}

$$
\left.\limsup _{m \rightarrow \infty} \iint Q|| P_{m}\right|^{2}-1 \mid \mathrm{d} \lambda \mathrm{d} \mathbb{P} \geqslant \iint Q \mathrm{~d} \lambda \mathrm{d} \mathbb{P} .
$$

Proof. We have

$$
\left.\iint Q|| P_{m}\right|^{2}-1\left|\mathrm{~d} \lambda \mathrm{d} \mathbb{P} \geqslant \int\right| \int Q\left(\left|P_{m}\right|^{2}-1\right) \mathrm{d} \mathbb{P} \mid \mathrm{d} \lambda .
$$

But, from (4.1)

$$
\int\left(\left|P_{m}\right|^{2}-1\right) \mathrm{d} \mathbb{P}=2 \operatorname{Re}\left\{\left(G_{p_{m}}\left(z^{h_{m}+t_{m}}\right)\right)\left(\int \tau_{1} \mathrm{~d} \mathbb{P}\right)\right\}+\left(\left|F_{p_{m}}\left(z^{h_{m}+t_{m}}\right)-\frac{p_{m}-1}{p_{m}}\right|\right) \phi_{m}(z) .
$$

Where, $F_{p}$ and $G_{p}$ is given, for any $p \in \mathbb{N}^{*}$, by 


$$
\begin{aligned}
& F_{p}(z)=\left|\frac{1}{\sqrt{p}} \sum_{k=1}^{p-1} z^{k}\right|^{2}, \\
& G_{p}(z)=\frac{1}{p} \sum_{k=1}^{p-1} z^{k} .
\end{aligned}
$$

Combine (6.1) and (6.2) to obtain

$$
\begin{aligned}
\left.\iint Q|| P_{m}\right|^{2}-1 \mid \mathrm{d} \lambda \mathrm{d} \mathbb{P} \geqslant & \iint Q\left(\left|F_{p_{m}}\left(z^{h_{m}+t_{m}}\right)-\frac{p_{m}-1}{p_{m}}\right| \phi_{m}(z)\right) \mathrm{d} \lambda \mathbb{P} \\
& -2 \iint Q\left|G_{p_{m}}\left(z^{h_{m}+t_{m}}\right)\right|\left|\int \tau_{1} \mathrm{~d} \mathbb{P}\right| \mathrm{d} \lambda \mathrm{d} \mathbb{P} .
\end{aligned}
$$

But, on one hand, we have

$$
\begin{aligned}
\int Q\left|G_{p_{m}}\left(z^{h_{m}+t_{m}}\right)\right|\left|\int \tau_{1} \mathrm{~d} \mathbb{P}\right| \mathrm{d} \lambda & \leqslant\left(\int\left|G_{p_{m}}\left(z^{h_{m}+t_{m}}\right)\right|^{2} \mathrm{~d} \lambda\right)^{1 / 2}\left(\int Q^{2} \mathrm{~d} \lambda\right)^{1 / 2} \\
& \leqslant\left(\int\left|G_{p_{m}}\left(z^{h_{m}+t_{m}}\right)\right|^{2} \mathrm{~d} \lambda\right)^{1 / 2}=\frac{1}{\sqrt{p_{m}}} \underset{m \rightarrow \infty}{\longrightarrow} 0 .
\end{aligned}
$$

On the other hand, since $\left|X_{m}\right| \leqslant t_{m}, \sum_{k \in X_{m}}\left(\xi_{m}\{k\}\right)^{2} \underset{m \rightarrow \infty}{\longrightarrow} 1$ and for any $f \in L^{1}$, we have

$$
\widehat{f_{(m)}}(n)= \begin{cases}0 & \text { if } n \text { is not divisible by } m, \\ \hat{f}\left(\frac{n}{m}\right) & \text { otherwise. }\end{cases}
$$

Where $f_{(m)}(z)=f\left(z^{m}\right)$, we get that

$$
\left|F_{p_{m}}\left(z^{h_{m}+t_{m}}\right)-\frac{p_{m}-1}{p_{m}}\right|\left|\sum_{k \in X_{m}} \xi_{m}(k) z^{k}\right|^{2} \mathrm{~d} \lambda
$$

converge to $K \cdot \lambda$, with $K \geqslant 1$. In fact

$$
\begin{aligned}
\int\left|F_{p_{m}}\left(z^{h_{m}+t_{m}}\right)-\frac{p_{m}-1}{p_{m}}\right|\left|\sum_{k \in X_{m}} \xi_{m}(k) z^{k}\right|^{2} \mathrm{~d} \lambda & =\sum_{k \in X_{m}}\left(\xi_{m}\{k\}\right)^{2} \int\left|F_{p_{m}}\left(z^{h_{m}+t_{m}}\right)-\frac{p_{m}-1}{p_{m}}\right| \mathrm{d} \lambda \\
& \geqslant \sum_{k \in X_{m}}\left(\xi_{m}\{k\}\right)^{2} \int\left(F_{p_{m}}\left(z^{h_{m}+t_{m}}\right)-\frac{p_{m}-1}{p_{m}}\right) z^{h_{m}+t_{m}} \mathrm{~d} \lambda \\
& =\sum_{k \in X_{m}}\left(\xi_{m}\{k\}\right)^{2}\left(\frac{p_{m}-2}{p_{m}}\right) \underset{m \rightarrow \infty}{\longrightarrow} 1
\end{aligned}
$$

and the lemma follows from (6.3).

Proof of Theorem 3.1 in the case of $\underline{\lim } \max _{s \in X_{m}} \xi_{m}(s)=1$. As in the case of $\underline{\lim } \max _{s \in X_{m}} \xi_{m}(s)<1$, we use the Lemma 6.1 to establish that

$$
\lim _{n \rightarrow \infty} \int \prod_{k=1}^{n}\left|P_{k}(z)\right| \mathrm{d} \lambda \mathrm{d} \mathbb{P}=0
$$

and the proof of Theorem 3.1. is complete.

Remark 6.2. It is an easy exercise to apply our machinery to the Rudolph construction in [18]. 


\section{Acknowledgements}

The authors would like to express thanks to J.-P. Thouvenot who posed them the problem of singularity of the spectrum of the Generalized Ornstein transformations.

\section{References}

[1] T.R. Adams, On Smorodinsky conjecture, Proc. Amer. Math. Soc. 126 (3) (1998) 739-744.

[2] T.R. Adams, N.A. Friedman, Staircase mixing, Ergodic Theory Dynam. Systems, in press.

[3] A. Bonami, Ensembles $\Lambda(p)$ dans le dual de $D^{\infty}$, Ann. Inst. Fourier (Grenoble) 15 (1968) 293-304.

[4] J. Bourgain, On the spectral type of Ornstein class one transformations, Israel J. Math. 84 (1993) 53-63.

[5] J.R. Choksi, M.G. Nadkarni, The maximal spectral type of rank one transformation, Canad. Math. Pull. 37 (1) (1994) 29-36.

[6] D. Creutz, C.E. Silva, Mixing on a class of rank one transformations, Ergodic Theory Dynam. Systems 24 (2) (2004) 407-440.

[7] R.M. Dudley, Real Analysis and Probability, Wadsworth \& Brooks/Cole Math. Ser., Wadsworth \& Brooks/Cole, Pacific Grove, CA, 1989.

[8] E.H. El Abdalaoui, La singularité mutuelle presque sûre du spectre des transformations d'Ornstein, Israel J. Math. 112 (1999) $135-155$.

[9] E.H. El Abdalaoui, On the spectrum of the powers of Ornstein transformations, Special issue on Ergodic theory and harmonic analysis, Shankyā Ser. A 62 (3) (2000) 291-306.

[10] N. Friedman, Replication and stacking in ergodic theory, Amer. Math. Monthly 99 (1992) 31-34.

[11] N.A. Friedman, Introduction to Ergodic Theory, Van Nostrand Reinhold, New York, 1970.

[12] I. Klemes, The spectral type of staircase transformations, Tohoku Math. J. 48 (1994) 247-258.

[13] I. Klemes, K. Reinhold, Rank one transformations with singular spectre type, Israel J. Math. 98 (1997) 1-14.

[14] M.G. Nadkarni, Spectral Theory of Dynamical Systems, Birkhäuser Advanced Texts: Basler Lehrbücher (Birkhäuser Advanced Texts: Basel Textbooks), Birkhäuser, Basel, 1998.

[15] D.S. Ornstein, On the root problem in ergodic theory, in: Proc. Sixth Berkeley Symposium in Math. Statistics and Probability, University of California Press, 1971, pp. 347-356.

[16] W. Parry, Topics in Ergodic Theory, Cambridge University Press, 1981.

[17] A.A. Prikhod'ko, Stochastic constructions of flows of rank 1, Sb. Math. 192 (11-12) (2001) 1799-1828.

[18] D. Rudolph, An example of a measure-preserving map with minimal self-joining and applications, J. Anal. Math. 35 (1979) $97-122$. 